\title{
Elektromagnetische Verträglichkeit und Robustheit elektronischer Systeme als Herausforderung des Technologiezeitalters
}

\author{
B. Deutschmann
}

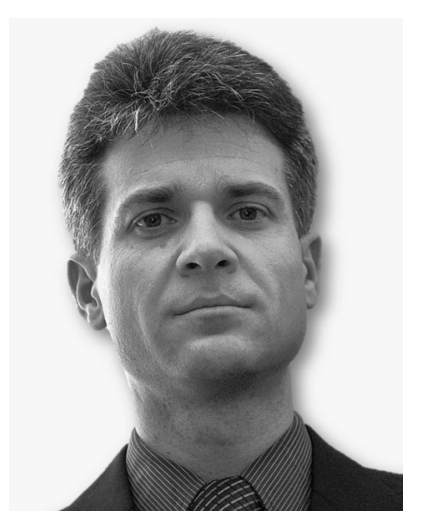

Univ.-Prof. Dipl.-Ing. Dr. Bernd Deutschmann

\begin{abstract}
Liebe Leserinnen, liebe Leser,

das Thema der elektromagnetischen Verträglichkeit (EMV) von elektronischen Systemen - nämlich Störfestigkeit auf der einen Seite und Störemission auf der anderen Seite - ist in der heutigen, in allen denkbaren Bereichen zunehmend technisierten Welt allgegenwärtig.
\end{abstract}

Vor gar nicht allzu langer Zeit bestand zum Beispiel die Elektronik in einem modernen Haushalt - eigent-

lich müsste man in diesem Zusammenhang eher von Haushaltselektrik sprechen - im Wesentlichen aus Beleuchtung, Elektroherd, Küchenmixer, Waschmaschine und Bügeleisen. Abgesehen von einem Rundfunkempfänger oder vielleicht sogar Fernsehapparat gab es kaum elektronische Einrichtungen, die durch elektromagnetische Störungen beeinflusst werden konnten.

Heute macht uns eine Vielzahl modernster elektronischer Systeme im Haushalt unser Leben leichter. Intelligente Steuerungen erlauben etwa von fast überall auf der Welt, das Licht im Haus ein- und auszuschalten, die Alarmanlage zu aktivieren und am Smartphone nachzusehen, wer in den letzten Stunden an der Tür geklingelt hat. Sogar unsere Heizung weiß, wann wir uns wieder auf dem Heimweg befinden und beginnt, selektiv einzelne Wohnräume aufzuheizen, wenn wir uns unserem Haus nähern. Das reibungslose Zusammenwirken der in ihrer Zahl und Komplexität zunehmenden elektronischen Systeme stellt aber auch sehr hohe Ansprüche an deren elektromagnetische Verträglichkeit. Insbesondere sollen sich die elektronischen Systeme nicht durch elektromagnetische Einwirkungen in ihrer Funktion beeinflussen lassen und auch nicht übermäßig viel Störemission an ihre Umgebung abgeben.

Was hier beispielhaft an der Haushaltselektronik aufgezeigt wurde, gilt auch für alle anderen Bereiche, in denen uns elektronische Systeme das Leben erleichtern sollen. Wo elektronische Systeme miteinander koexistieren müssen, sind Themen wie deren Robustheit gegen elektrostatische Entladungen (ESD) und Radio Frequency Interferences (RFI), funktionale Sicherheit, Zuverlässigkeit und elektromagnetische Verträglichkeit nicht mehr wegzudenken. Werden elektronische Systeme zur Unterstützung des Menschen eingesetzt, sind diese Themen sogar sehr oft von entscheidender Bedeutung, da bei einer Fehlfunktion eine unmittelbare Gefährdung von Personen entstehen kann.
Unverzichtbar ist dabei eine frühzeitige Einbeziehung und Betrachtung dieser Anforderungen in die Konzept- und Designphase der elektronischen Systeme, denn deren elektromagnetische Verträglichkeit ist meist nur durch vorbeugende Berücksichtigung von Projektbeginn an erreichbar - und zwar durch Einbeziehung der EMV-Anforderungen auf allen Ebenen: integrierter Schaltkreis - elektronische Komponente - Gesamtsystem. Den integrierten Schaltkreisen kommt dabei eine Schlüsselrolle zu, da diese das EMVVerhalten des späteren Endprodukts wesentlich beeinflussen können. Eine zu geringe Störfestigkeit, beziehungsweise eine zu hohe Störemission der eingesetzten integrierten Schaltkreise, birgt das Risiko von Fehlfunktionen des gesamten Systems in sich.

Die Beiträge dieses Schwerpunktheftes stellen einen exemplarischen Streifzug durch wichtige Forschungsaktivitäten der Industrie, von Forschungseinrichtungen und der Institute für Elektronik, für Hochspannungstechnik und Systemmanagement sowie für Hochfrequenztechnik der TU Graz zu dieser Thematik dar. So finden sich in dieser Ausgabe Beiträge, die sich mit dem Thema der elektromagnetischen Verträglichkeit beschäftigen, aber auch Beiträge zum Thema Robustheit an sich.

Da elektrostatische Entladungen, wie sie zum Beispiel bei atmosphärischen Entladungen auftreten können, oft als Ursache für Fehlfunktionen und Systemausfälle gesehen werden, besteht speziell im Bereich der Mikroelektronik akuter Bedarf an Verfahren, die Störfestigkeit eines neuen Produktes schon während der Konzept- und Designphase, aber auch entwicklungsbegleitend zu bestimmen. Diesem Thema widmet sich ein Beitrag des Instituts für Elektronik in Kooperation mit dem Institut für Hochspannungstechnik und Systemmanagement der TU Graz, welcher die Zusammenhänge zwischen atmosphärischen Entladungen in Verbindung mit elektrischen Systemen, elektronischen Geräten und integrierten Schaltungen aufzeigt. Dabei wird auch auf die Prüfverfahren und Normen zur Sicherstellung der elektromagnetischen Verträglichkeit gegenüber transienten Störgrößen eingegangen

In diesem Zusammenhang ist es natürlich auch sehr wichtig, die verbauten elektronischen Komponenten und speziell die integrierten Schaltkreise gegen auftretende transiente Störungen zu schützen. Ein Beitrag der Infineon Technologies AG/München behandelt dieses Thema und gibt einen Überblick über Transient Voltage Suppressors sowie deren Technologie und Anwendungen.

Solche Schutzstrukturen werden oft in der Nähe von Steckkontakten auf Leiterplatten verbaut. In einem weiteren Beitrag des Instituts für Elektronik wird gezeigt, wie eine so genannte "Surface Scan Methode" verwendet werden kann, um die Ausbreitungswege von

Deutschmann, Bernd, Institut für Elektronik, Technische Universität Graz, Inffeldgasse 12/I, 8010 Graz, Österreich (E-Mail: bernd.deutschmann@tugraz.at) 
transienten Störströmen auf einer Leiterplatte zu visualisieren. Solche transiente Störungen können zum Beispiel durch eine elektrostatische Entladung beim Berühren der Steckkontakte der Leiterplatte verursacht werden.

Dieses Messverfahren wurde auch zur Untersuchung der elektromagnetischen Störabstrahlung eines intelligenten drahtlosen Temperaturüberwachungssystems verwendet, welches in einem Beitrag der Infineon Technologies AG/Graz vorgestellt wird.

Besonders bedeutsam ist, dass nicht nur die einzelnen verbauten Schaltkreise, sondern alle elektronischen Systeme den normativen EMV-Anforderungen genügen. Selbst wenn alle einzelnen elektronischen Komponenten EMV-konform sind, kann es immer noch vorkommen, dass das Endprodukt nicht robust genug ist. Dieses spannenden Themas nimmt sich beispielsweise ein in diesem Schwerpunktheft enthaltener Beitrag der Seibersdorf Labor GmbH an - zu finden in der Rubrik "praxis+wissen" dieser Ausgabe an.

Ein weiterer Beitrag, der durch Zusammenarbeit der Institute für Elektronik und Hochfrequenztechnik entstand, widmet sich dem Thema der Prüfung der elektromagnetischen Verträglichkeit von analogen Funkmikrofonsystemen. Die Autoren beschreiben dabei das komplette Messverfahren mit zwei Messaufbauten, die Prüfung mit Störsignalen mit unterschiedlichen Modulationsarten (AM, FM und DVB-T) sowie die Überprüfung der Qualität des übertragenen Audiosignals mittels Total Harmonic Distortion + Noise-Messungen.

Die Verträglichkeit beziehungsweise Robustheit eines elektronischen Systems muss aber nicht nur dessen Störfestigkeit gegenüber Störsignalen wie transienten Störungen oder Radio Frequency interference (RFI) bedeuten. Daher wurde für dieses Schwerpunktheft bewusst auch das immer wichtiger werdende Thema "Radiation Hardness" miteinbezogen. Diesem Thema widmen sich gleich drei Beiträge. Im Beitrag des Instituts für Elektronik werden die Effekte der ionisierenden Strahlung auf integrierte Schaltungen beschrieben und ein Überblick über aktuelle Techniken zur Reduktion ihrer Auswirkungen aufgezeigt. Der erste der beiden weiteren Beiträge der Seibersdorf Laboratories beschreibt die Schädigung von
Power-MOSFETs in ionisierenden Strahlungsfeldern und zeigt die Degradierung wesentlicher Bauteilparameter sowie die Anfälligkeit der Power-MOSFETs für Single Event Gate Rupture (SEGR) bei Exposition in Schwerionenfeldern. Der zweite Beitrag beschreibt die neue 60Co-Anlage zum 24/7-Testen von elektronischen Komponenten und Geräten in Bezug auf Strahlungsfestigkeit gegenüber ionisierender Strahlung.

Zum breiten Feld der elektromagnetischen Verträglichkeit elektronischer Systeme gehört neben der Störfestigkeit auch das Thema der Störemissionen. Ein Beitrag des Instituts für Elektronik behandelt dazu die Möglichkeiten zur Reduzierung der Störemissionen von Class-D-Audioverstärkern mittels Spread Spectrum-Techniken. Da gerade die vermehrt in mobilen Geräten verwendeten ClassD-Audioverstärker im Vergleich zu anderen Verstärkern aufgrund ihrer Schaltaktivität meist sehr hohe Störemissionen erzeugen, ist die Spread Spectrum-Technik eine vielversprechende Methode, um Emissionen entsprechend zu reduzieren.

Es sei an dieser Stelle angemerkt, dass die Beiträge in diesem Schwerpunktheft der e\&i nur einen exemplarischen Überblick in Form von ein paar wichtigen Themen geben können. Für eine umfassende Information über die Aktivitäten im Bereich der elektromagnetischen Verträglichkeit wird der Besuch der EMV-Fachtagung empfohlen. Diese Veranstaltung, die am 12. und 13. April 2016 in Seibersdorf nunmehr zum 14. Mal stattfindet, hat sich im Laufe der Zeit zu einer bekannten Branchenplattform für die Industrie und akademische Institutionen in Österreich entwickelt.

Ich möchte mich abschließend noch ganz herzlich bei den Autoren der vorliegenden Beiträge sowie bei den Reviewern für deren professionelle Gutachten bedanken.

Ihnen, liebe Leserinnen und Leser, hoffe ich, mit diesem Schwerpunktheft zum Thema Robustheit, elektrostatische Entladungen und elektromagnetische Verträglichkeit einen informativen Überblick zu geben und freue mich sehr, wenn es mir gelungen ist, die Begeisterung für dieses Thema nach außen zu tragen.

Ich wünsche Ihnen viel Vergnügen beim Lesen der Beiträge! 